\title{
NEURODEVELOPMENTAL CARE OF PRETERM INFANTS AND ITS KEY ELEMENTS
}

\author{
I. M. Sarapuk', H. A. Pavlyshyn', L. Lacina ${ }^{2}$, B. Królak-Olejnik ${ }^{3}$ \\ I. HORBACHEVSKY TERNOPIL STATE MEDICAL UNIVERSITY, TERNOPIL, UKRAINE' \\ NIDCAP CINCINNATI TRAINING CENTER, CINCINNATI CHILDREN'S HOSPITAL \\ MEDICAL CENTER, CINCINNATI, OHIO, USA ${ }^{2}$ \\ WROCLAW MEDICAL UNIVERSITY, WROCLAW, POLAND
}

\begin{abstract}
During the past few decades, advancements in the perinatal and neonatal intensive care have led to a significant survival of premature infants. However neurodevelopmental outcomes remain the major issues of concern. Developmental care is an approach that aims to reduce the mismatches between the extra- and intrauterine environment, decrease the stress of preterm newborns in neonatal intensive care units, and thus promote optimal neurobehavioral development of the infant.

The Newborn Individualized Developmental Care and Assessment Program (NIDCAP) model was developed as a clinical framework for the implementation of developmental care. The model focuses on detailed reading of each individual infant's behavior. By observing the infant during a routine assessment (before, during and after) and a detailed description of his/her behavioral responses, a professional can assess the ability of the infant's immature nervous system to tolerate the environment and care manipulations. Such evaluation will enable one to determine the adequacy of the environment and care to meet the infant's opportunities and needs, with subsequent corrections and adaptation. NIDCAP's aim is to support the infant in his/her interaction with the environment as an active participant in caregiving within a family.

With the help of NIDCAP approaches in neonatal care medical staff will study how to read and interpret infants' behavior, thus hearing their voices.
\end{abstract}

KEY WORDS: developmental care, NIDCAP, preterm infants.

\section{Introduction}

Today the number of premature births is increasing. According to the World Health Organization (2012), every year about 15 million babies are born prematurely - more than one in 10 of all babies born around the world, affecting the families around the world. In developed countries of Europe and USA the incidence varies from 5 to $12 \%[1,2,3]$, and may reach $40 \%$ in less developed regions [2].

During the past few decades, advancements in the perinatal and neonatal intensive care (the use of antenatal corticosteroid therapy, surfactant therapy, and improved ventilatory techniques) have led to a significant survival of premature infants with gestational age less than 32 weeks [4]. The percentage of newborns with gestational age less than 32 weeks (extremely preterm and very preterm ones) is $1-2 \%$ in developed countries [1].

Corresponding author: Iryna Sarapuk, Department of Pediatrics №2, I. Horbachevsky Ternopil State Medical University, 2 Sakharova Street, Ternopil, Ukraine, 46400 Phone number: +380502081147

E-mail: prostoirusya@ukr.net
However, despite the positive results of survival, morbidity and complications associated with premature birth are growing rapidly $[2,3]$. Preterm babies meet with a large number of negative factors, since the beginning of their extrauterine life passes in the NICU (neonatal intensive care unit) environment, where such factors as light, noise, highly advanced noninvasive and invasive manipulation and pain dramatically affect the whole immature organism, resulting in early short-term and long-term complications.

Neurodevelopmental outcome has become a benchmark in determining the effectiveness of medical neonatal care [5]. The incidence of disability and neurodevelopmental problems among survivors of neonatal intensive care remains high and problematic $[2,6,7]$. Severe neurological complications (cerebral palsy, moderate to severe mental retardation, epilepsy, poor motor skills, sensorineural hearing loss, blindness) still remain consistently high. Moreover, by the age of 8 years, over $50 \%$ of very low birth weight preterm children require special 
educational services and $15 \%$ have repeated at least one grade in school $[3,8,9]$.

However, these are not the only neurological problems. The implementation of extended follow-up monitoring, using modern methods and scales of neurological evaluation allow us to identify мовшіre and more neuro-developmental disorders in children. These disorders include learning disabilities, low average IQ scores, attention deficit, hyperactivity disorders, neuropsychological deficits, visual motor disintegration, executive function difficulty, varying temperament difficulties, language delays, emotional problems and regulatory disorders $[5,8]$. $50-70 \%$ of very low birth weight premature infants suffer from these dysfunctions $[3,10]$.

In order to provide not only survival, but to optimize long-term health and development, it is necessary to know the features of brain development of the fetus in the intra-uterine environment and of the premature baby in the extrauterine space. The development of the brain of the full-term infant takes place in utero where the infant resides in a temperature-stable environment, receives all the nutrients, undergoes circadian rhythm, and most importantly, feels maternal presence and permanent protection $[11,12]$. Prematurely born infants are deprived of all these favorable conditions in the most critical period when their brain is growing and developing most intensively [13]. At the same time, they are exposed to excessive manipulation in a high-technology medical NICU environment. The mismatch between the preterm infants' need that are necessary for brain development, and the realities of intensive care units may cause serious problems of neuro-physiological, psycho-emotional and psychosocial development.

Developmental care is an approach that uses a range of medical and nursing interventions that aim to reduce the mismatches between extra- and intra-uterine environment, decrease the stress of preterm neonates in NICUs, and thus promote optimal neurobehavioral development of the infant.

\section{Review}

The framework of developmental care views preterm infants as fetuses who find themselves too early and unexpectedly in a technologic hospital environment instead of the evolutionarily promised mother's womb [3, 14]. Behavioral individuality of each infant is the basis of neuro-developmental care. Each infant is seen as an active participant in all elements and at all stages of care. An infant's behavior provides the best information to determines the care management $[14,15]$.

The Newborn Individualized Developmental Care and Assessment Program (NIDCAP) model was developed as a clinical framework for the implementation of developmental care. NIDCAP is a comprehensive program which includes a behavioral observation methodology and creation of individual developmental caregiving support of the infant's own developmental goals [12]. The model focuses on detailed reading of each individual infant's behavior [2]. By observing the child during a routine assessment (before, during and after) and a detailed description of his/her behavioral responses, medical staff can assess the ability of the infant's immature nervous system to tolerate the environment and care manipulations. Such evaluation will enable one to determine the adequacy of the environment and care to infant's opportunities and needs, with subsequent corrections and adaptation $[2,15]$.

The infant's ability to regulate and control the behavior occurs within continuous interaction with the environment (whether womb, NICU, home conditions) and is expressed through the infant's five systems: autonomic (physiological), motor, state, attention/ interaction and self-regulation. These systems are continuously interacting with one another. Each system influences and supports the other, and interacts with the environment. The autonomic system functioning is manifested in an infant's breathing quality and rate, color fluctuation, and visceral stability or instability. The functional state of motor system is estimated by the position, muscle tone and movement patterns of the face, trunk and extremities. State organization is observable in an infant's range of available levels of consciousness, their robustness and modulation of the available states, and the patterns of transition from state to state. The activity of attention/interaction system is manifested by infant's look, facial expressions and the ability to interact $[3,12,15]$. The self-regulation system is manifested in infant's ability to re-balance and bring into harmony the other systems when they have moved out of balance [2].

NIDCAP methodology was founded by Heidelise Als (psychologist from the Harvard University) in 1986 and is based on the Synactive Theory of Development. Heidelise Als has been a pioneer in helping medical staff and parents to understand how to "read" preterm infant's 
behavior, especially those who need intensive care [16].

The synactive theory of newborn behavioral organization and development (synaction n., or synactive adj. (from the Greek syn "together" and the Latin actio "action," resulting in "together in action"). The synactive theory of newborn behavioral organization and development suggests that development of the human fetus, and later newborn, proceeds through the constant balancing of approach and avoidance behaviors, leading to a continuous interaction of the above mentioned subsystems and their increasingly defined delineations within the organism, and the organism's interaction with the environment at large $[12,17]$.

The synactive theory of infant development provides that there is no possibility for development without stimulation, but there is also no harmonious development if the stimuli lead to disorganization and stress [15]. Sensory inputs are the important and necessary parameters in fostering central nervous system development [3]. Inappropriate stimuli penetrate into all systems and destroy them, while adequate and timely stimuli promote and improve the infant's growth and development $[11,15]$. The infant's response to any stimulus manifests in signs of adaptation of the foregoing systems when he or she tolerates it, or in maladaptive signs if the infant is unable to cope. The infant is able to tolerate stimuli when they are appropriate in timing, complexity and intensity in relation to the infant's thresholds of functioning. If the stimuli are too intense, complex or inappropriately in time, infant either has strategies to move away from inputs, avoid them, or suffer from them. Such behavior is considered a stressful situation for an infant $[2,12]$. The aim of the NIDCAP is to support the infant in his/her growing tolerance to stimuli, and to minimize stressful events and manipulations that require energy consumption, use of calories and sometimes break the physiological homeostasis [3]. Each caregiver must recognize that for the unstable or fragile infant the best form of sensory input may necessitate a plan of reduction of surrounding stimuli [18].

Signs of autonomic system adaptation in the infant are: stable color, regular breathing and heart rate within physiological ranges and stable digestion. Self-regulatory balance of motor system is reflected by the appropriate muscle tone, harmonious and smooth movements, softly flexed posture with flexed arms and legs; hands near the face, around the mouth or inside it [12,
15]. Sleep and waking cycles develop in time to become differentiated $[15,19]$. The infant actively looks for a source of stimulation and visual contact with a person who cares for him/ her, the face is open, and the infant has attentive look. This stability of the systems manifests in smooth function and reflects intact organization and central nervous system control [3].

Signs of autonomic system stress in the infant are: skin discoloration (pale, marble, red, cyanotic), breathing disorders (apnea, tachypnea) with or without desaturation, unstable heartbeat (bradycardia or tachycardia), functional gastrointestinal disorders (hiccups, regur_ gitation, vomiting) and neurological disorders (tremors, convulsions, yawning, sighing). Motor signs of stress include: muscle hypotonia, extension of the trunk (arching), ourward movements of limbs (splaying, airplanning, saluting, sitting on air) and tongue extensions. Sleep and waking cycles are not proper differentiated, with the infant avoiding stimulation and visual contact, there is lack of visual concentration. In general, extension behaviors are thought to reflect stress, and flexion behaviors are thought to reflect self-regulatory competence. Diffuse behaviors are thought to reflect stress, and well-defined behaviors are thought to reflect regulatory balance $[12,15]$. Such stress reactions may indicate that environmental stimuli of light or noise or even caregiving events such as turning the infant or changing the diaper, may be overwhelming [3]. To overcome these stressful situations infant spends the energy that he needs to maintain his homeostasis $[3,15]$.

Another behavioral scenario is possible when the infant cannot respond to environment or handling stimuli. The infant may lie in the bed, limp and flaccid, unable to put any energy into responding to caregiver; or just develop minimal ranges of responsiveness $[15,17]$.

The ability to regulate or control the infant's autonomic, motor and state organization is a requirement of early development and it is called self-regulation [20]. Early self-regulation is accomplished in the mother's body as a fetus, and continues over the course of the first three years of life after birth. The first tasks of selfregulation involve the regulation of physiological function as breathing, heart rate regulation, maintenance of visceral control, body temperature and homeostasis and day-night cycles regulation. Later the infant learns to calm himself and relax even after mild stress. As an infant matures in the context of daily interactive care from consistent primary caregivers, self regula- 
tion becomes the task of learning to control motor behaviors, sleep-wake cycles, attention and interaction $[3,15,20,21]$. Eventually, these capacities merge into emotional regulation. The premature baby often needs external assistance in self-regulation. Parents and caregivers become the infant's "co-regulators" and support infants by accurately reading and interpreting the infant's behavioral signals [3].

How assess and interpret the child's behavior according to NIDCAP? Developmental specialists observe an infant's behavior weekly or every other week throughout the hospitalization, starting with the phase of the infant's initial stabilization. During each observation, the developmental specialist systematically records an infant's behavior for approximately 20 minutes before a planned medical or nursing caregiving interaction and continues to observe throughout the duration of the interaction and for approximately 20 minutes after it. Ninetyone behaviors, including autonomic (breathing, heart rate, color changes and visceral signs), motor (postures, muscle tone fluctuations and movements) and state organization behaviors (levels of arousal, patterns of transitions between states, and clarity and robustness of sleep and awake states) are monitored every 2 minutes. Behaviors are conceptualized as regulatory and as stress according the foregoing signs of adaptation and disadaptation and are interpreted as indices of the infants' current strength and vulnerabilities respectively [14].

After the observation the developmental specialists write descriptive neurobehavioral reports and suggestions, which describe the infant's strengths, current sensitivities and apparent goals and thresholds to stress, and the infant's self-regulatory efforts at rest and during care manipulations. It helps to structure caregiving procedures to the infant's sleep/ wake cycle, adapt or modify the environment and care according to the child's needs with the aim to maintain the infant's well-regulated behavioral balance in an effort to promote the infant's strengths and simultaneously to reduce the infant's self-regulatory vulnerability $[2,14]$. As the infant matures, these recommendations are modified appropriately [22].

Key elements for implementation of individualized developmental family-centered care NIDCAP : 1) provision of sensitive care and handling for baby, 2) creation of a calm NICU environment, 3) parental involvement in infant care, their support, 4) presence of infant developmental specialist [3].
Provision of sensitive care and handling for the infant based on an accurate reading of infant behavioral signals and respect for the message that the infant communicates, along with recognition that the infant actively participates in all aspects of neonatal care [3].

a) Structure the infant's 24-hour day in accordance with the individual infant's sleep-wake cycles, state of alertness, medical needs and feeding competence. It may be necessary to divide or to combine procedures (eg. medical examination and care manipulations), to offer pauses according to the child's behavioral responses or to perform care with two persons. This approach provides infant's tranquility and thus promotes his/her growth [15].

b) Non-nutritive sucking suppors infant's physiological stability, improves sleep/waking cycle and reduces time of hospitalization [23].

c) Infant's flexed position reduces stress reactions, improves self-regulation, physiological stability and sensory stimuli tolerance and prevents the skeletal deformities [24, 25].

d) Positioning in the "nests" provides flexed position of the whole body with pelvic support (physiological position of the fetus), which prevents the formation of pathological extension position and hypertonus $[24,25]$.

e) Swaddling reduces physiological distress, maintains temperature homeostasis, improves motor development and optimizes the sleep/ waking cycle [26].

Creation of a calm NICU. It is necessary to create a supportive, gentle environment for the most fragile infants (pic. 1, 2)

a) Noise reduction (reduction of alarm volume, decreased ringtone volume of telephones, modification of individual and collective behaviors - quiet conversation, avoiding the noisy gestures, individual rooms, installation of a sound measurement device. Sound levels shall

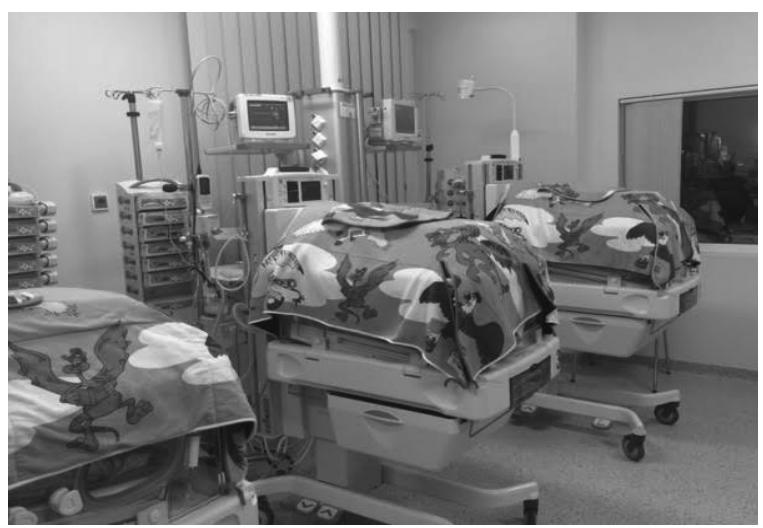

Pic. 1. Incubators in the NICU are covered to reduce the light and noise, to simulate day-night cycle. 


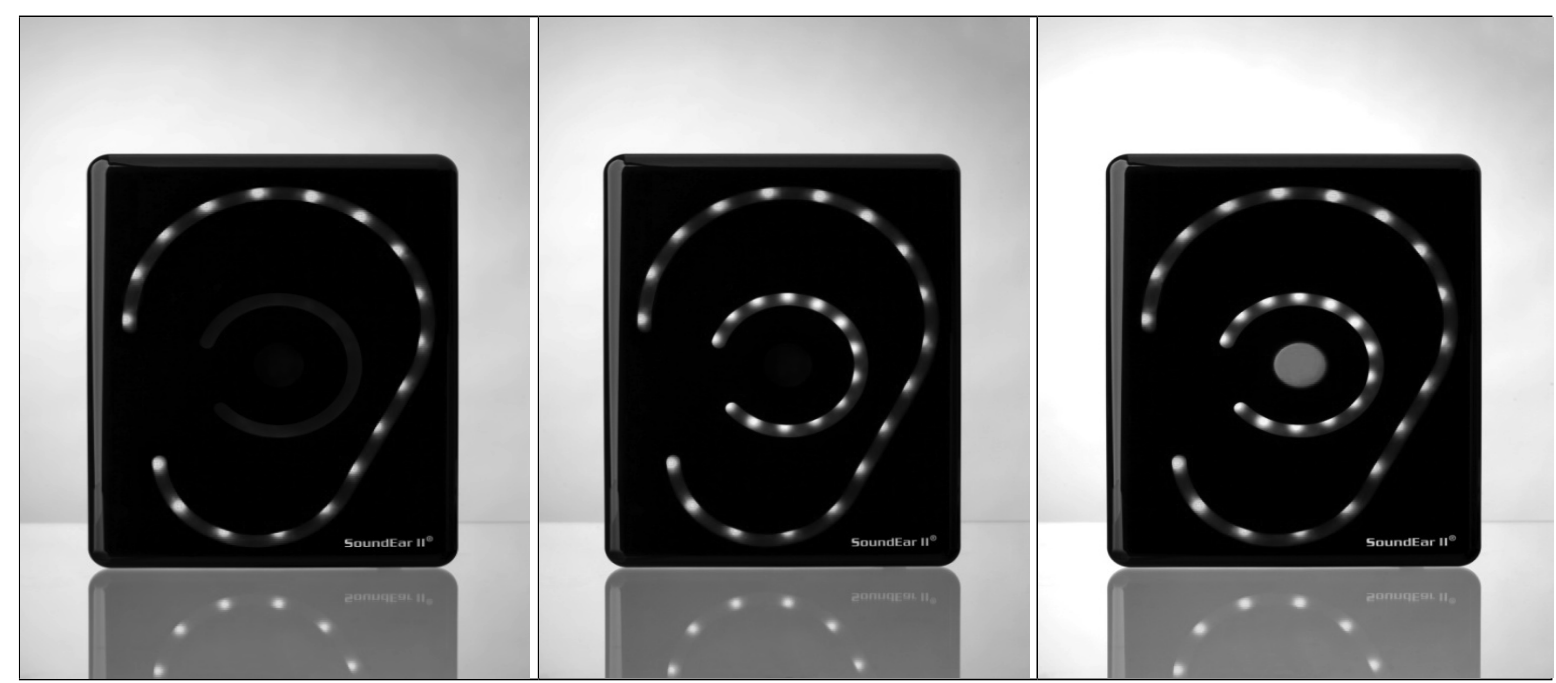

Pic. 2. The device for monitoring the volume of sounds in hospital wards (green - normal, yellow - loud, red - noice).

not exceed $45 \mathrm{~dB}$ and hourly $\mathrm{L} 10$ of $55 \mathrm{~dB}$; transient sounds should not exceed $70 \mathrm{~dB}$; equipment in NICU should have noise levels of $40 \mathrm{~dB}$ $[27,28]$.

b) Reduction of the light level (avoiding direct illumination into the infants'eyes, except during examination, incubator cover adapted to the infant's awakening, individual illumination lamps at each incubator, providing circadian rhythm with decreasing light intensity at night, access to daylight) $[3,27]$.

Parental involvement in infant care, their support.

a) Early parental involvement in caregiving and participation in all care with day-and-night access in the NICU [15].

b) Skin-to-skin contact with mother or father, including children who need invasive and non-invasive respiratory support. Such contact has significant advantages for both the infant (temperature homeostasis, respiratory stability with a decrease in apnea and brady-

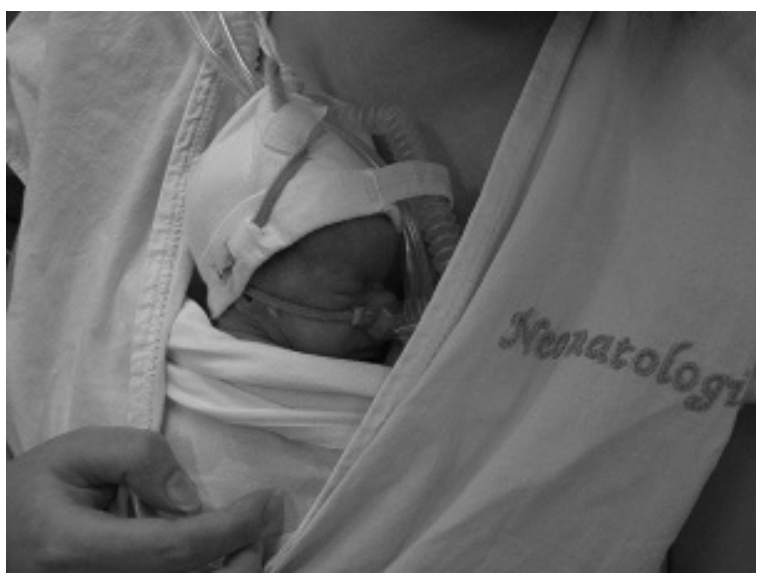

Pic. 3. Kangaroo mother care ("skin-to-skin"contact) of the preterm infant with nCPAP. cardia, calm and lasting sleep, feeling of safety, positive dynamics of body weight, decreased risk of nosocomial infections) and mother (improving of lactation, stress reducing, sense of paternity) $[15,29,30]$, (pic. 3, 4, 5, 6, 7).

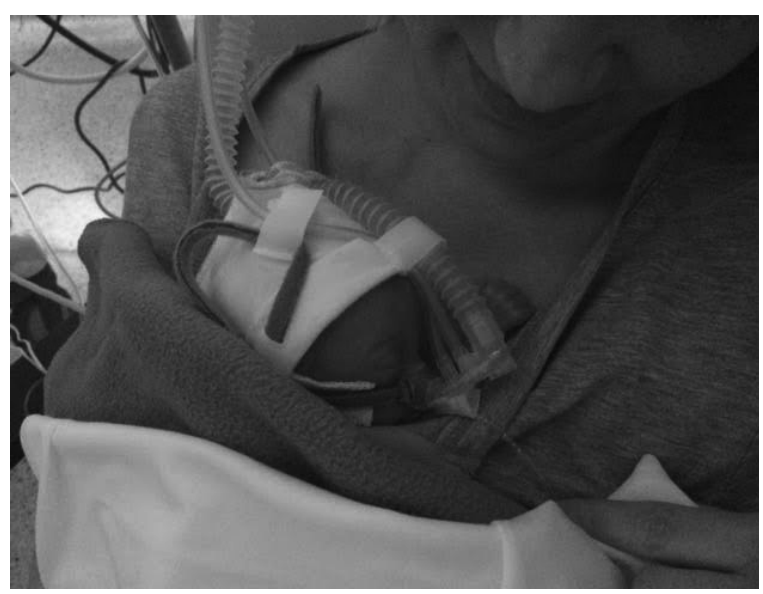

Pic. 4. Kangaroo mother care of the ELBW infant with nCPAP.

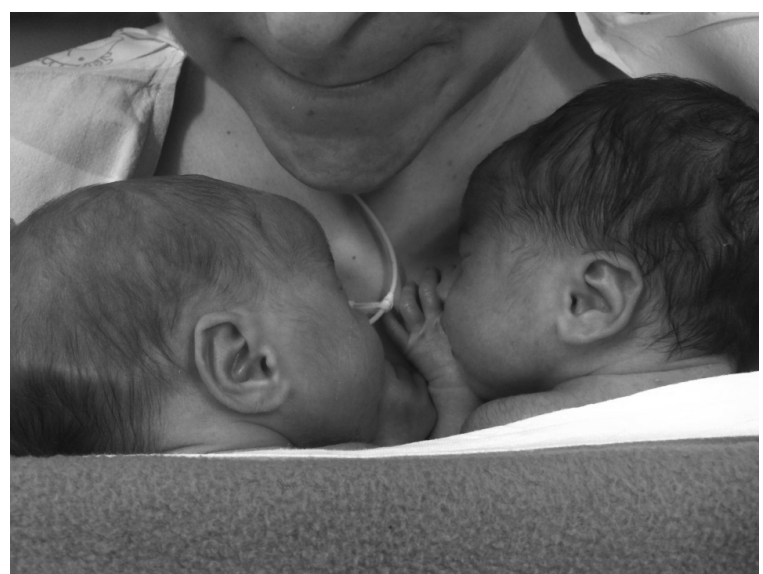

Pic. 5. Kangaroo mother care of the twins. 


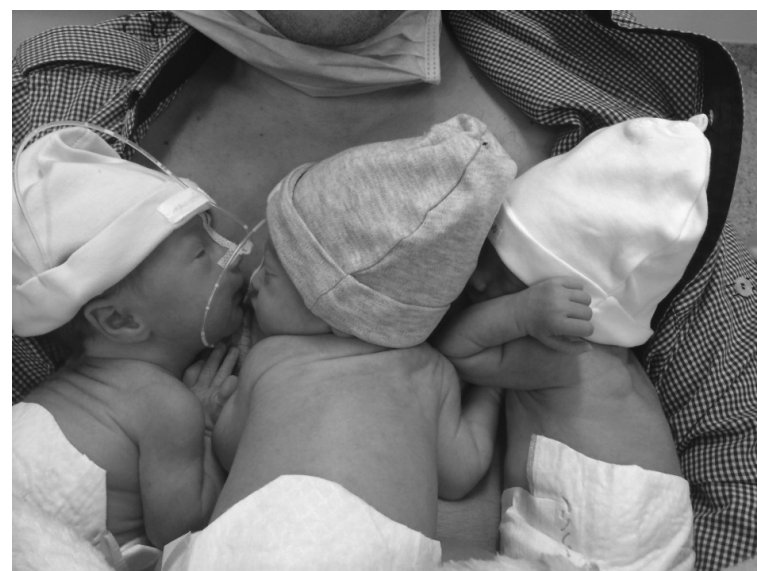

Pic. 6. Kangaroo father care of triplets.

Developmental care supports the relationship between the child and the family [12]. For the human species, parents are the familiar, constant and steady connection in an infant's life. They assure their infant's safety, and assure their child's development of trust and as a whole person [2]. The core principle of NIDCAP is the collaboration with each infant and the infant's parents as the primary caregivers and co-regulators; and the main goal of developmental care in the NICU and throughout the hospital stay is to improve child and family comfort and outcomes.

The presence of infant developmental specialist. The developmental specialist conducts regular NIDCAP observations and staff/parent teaching, provides consistency of developmental support to medical staff, family and the infant, articulates and adjusts the NICU

in the accordance with the developmental needs of the infant [3].

Positive aspects of developmental care. The studies have documented the beneficial effect of NIDCAP in terms of shorter intensive care and overall hospital stay, decreased time on the ventilator, decreased oxygen days, shorter duration of parenteral feeding, shorter transition periods to full enteral feeding, better average daily weight gain, lower total hospital charges and effect in enhancing neurodevelopmental outcome [11, 31-4]. Some research has also documented that the NIDCAP approach enhances brain structure and function

\section{References}

1. Ancel PY, Goffinet F, EPIPAGE 2 Writing Group. EPIPAGE 2: a preterm birth cohort in France in 2011. BMC Pediatr. 2014;14:97.

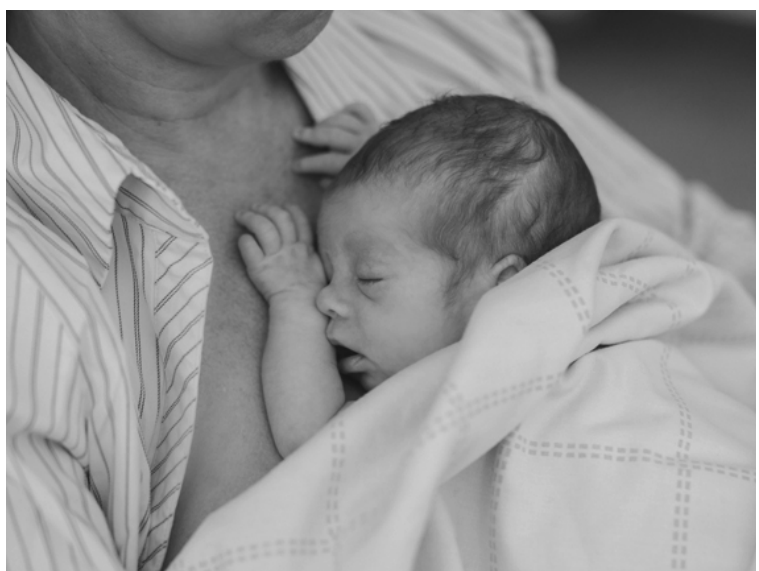

Pic. 7. Relaxing in skin-to-skin contact after breast-feeding.

when measured by sophisticated medical techniques such as EEG and MRI [35].

NIDCAP also has significant benefits for parents. The studies of $\mathrm{H}$. Als and other developmental specialists show the reduction in parenting stress and enhanced parent perception of the infant $[31,36,37]$. Except for shortterm positive effects, neonatal care according the NIDCAP has also long-term effects on the infant's neurological development, behavior and mother-child interaction $[34,36]$.

\section{Conclusions}

As survival of high risk infants has continued to improve, greater emphasis has now been placed on improving neurological outcomes and quality of life. The implementation of individualized developmental care encourages the shift from a protocol-based task and schedule oriented framework of NICU care to an individualized and relationship-based framework of care. The introduction of NIDCAP in the neonatal departments will reduce the mismatch between intra-uterine environment and NICU by taking into account the individual infant's current thresholds of behavioral organization, diminishing stress, and supporting each infant's strengths and competencies. With the help of NIDCAP approaches in neonatal care all staff will study how to read infants' behavior, to hear their voices and understand them. Our smallest and most fragile patients should feel comfort and a sense of security that are so important for their healthy development.

2. Als H, McAnulty GB. The Newborn Individualized Developmental Care and Assessment Program (NIDCAP) with Kangaroo Mother Care (KMC): 
Comprehensive Care for Preterm Infants. Curr Womens Health Rev. 2011;7(3):288-301.

3. VandenBerg KA. Individualized developmental care for high risk newborns in the NICU: A practice guideline. Early Human Development. 2007;83:433442.

4. Khan MR, Maheshwari PK, Shamim H, Saleem AF, Shakeel A, Syed RA, et al. Neurodevelopmental Outcomes of Premature Infants at a Tertiary Care Center in Pakistan. Pediatric Neurology. 2012;47:109-113.

5. Aylward GP. Neurodevelopmental outcomes of infants born prematurely. J Dev Behav Pediatr. 2005; 26:427-40.

6. Hack M, Flannery DJ, Schluchter M, Cartar L, Borawski E, Klein E. Outcomes in young adulthood for very low birth weight infants. N Engl J Med. 2002;346: 149-57. www.ncbi.nlm.nih.gov/pubmed/11796848. Accessed 17 Jan 2002.

7. Saigal S, den Ouden I, Wolke D. School-age outcomes in children who were extremely low birth weight from four international population-based cohorts. Pediatrics. 2003;112:943-50.

8. Rushing S, Ment LR. Preterm birth: a cost benefits analysis. Semin Perinatol. 2004;28:444-50. www. ncbi.nlm.nih.gov/pubmed/15693401. Accessed Dec 2004.

9. Bennett FC. Developmental outcome (in) MacDonald MG, Seshia MK, Mullett MD (eds.) Avery's Neonatology: Pathophysiology and management of the newborn. 6th edition. New York: Lippincott Williams and Wilkins; 2005:1632-52.

10. Hellstrom-Westas L, Roson I. Continuous brain-function monitoring: State of the art in clinical practice. Semin Fetal Neonatal Med. 2006;11(6): 503-11.

11. Als $\mathrm{H}$, Lawhon $\mathrm{G}$. Theoretical perspective for developmentally supportive care (in) Kenner C, McGrath JM (eds). Developmental care of newborns and infants: A guide for health professionals. St. Louis: Mosby; 2004:47-58.

12. Als H. and Gilkerson L. The role of relationshipbased developmentally supportive newborn intensive care in strengthening outcome of preterm infants. Seminars in Perinatology. 1997;21(3):178-189. www. ncbi.nlm.nih.gov/pubmed/9205974. Accessed Jun 1997.

13. McLennan JE, Gilles FH, Neff R. A model of growth of the human fetal brain (in) Gilles $\mathrm{FH}$, Leviton A, Dooling EC (eds). The developing human brain. Boston: John Wright; 1983:43-59.

14. Als H, Duffy FH, McAnulty GB, Rivkin MJ, Vajapeyam $\mathrm{S}$, Mulkern RV, et al. Early experience alters brain function and structure. Pediatrics. 2004;113(4): 846-57.

15. Als $H$. Reading the premature infant (in) Goldson E (ed.) Developmental interventions in the neonatal intensive care nursery. New York: Oxford University Press; 1999:18-85.

16. LaRossa MM. Understanding Preterm Infant Behavior in the NICU. 2017. http://www.pediatrics. emory.edu/divisions/neonatology/dpc/nicubeh.html. Accessed 2017.
17. Als H. Toward a synactive theory of development: Promise for the assessment of infant individuality. Inf Mental Health J. 1982;3:229-43.

18. Holditch-Davis D, BlackburnST, VandenBerg KA. Newborn and infant neurobehavioral development (in) Kenner C, Lot JW (eds). Comprehensive neonatal nursing: A physiologic perspective. 3rd edition. Philadelphia: Saunders; 2003:236-84.

19. Brazelton TB. Behavioral competence (in) Avery GB, Fletcher MA, MacDonald MG (eds.) Neonatology: Pathophysiology and management of the newborn. Philadelphia: Lippincott Williams and Wilkins; 1999:321-7.

20. Shonkoff J, Phillips D. From neurons to neighborhoods: The Science of early childhood development. Wash DC: National Academy Press; 2000:93-124.

21. Schore AN. Affect dysregulation and disorders of the self. J Can Acad Child Adolesc Psychiatry. 2006;15(2):100-101.

22. Bjorn Westrup. Newborn Individualized Developmental Care and Assessment Program (NIDCAP) Family-centered developmentally supportive care. Early Human Development 2007;83:443-449.

23. Foster JP, Psaila K, Patterson T. Non-nutritive sucking for increasing physiologic stability and nutrition in preterm infants. Cochrane Database of Systematic Reviews. 2016;10. http://www.cochrane. org. Accessed 25 Feb 2016.

24. Als H, Lawhon G, Duffy FH, McAnulty GB, GibesGrossman R, Blickman JG. Individualized developmental care for the very low-birth-weight preterm infant. Medical and neurofunctional effects. JAMA. 1994;272(11):853-8.

25. Monterosso L, Kristjanson L, Cole J. Neuromotor Development and the Physiologic Effects of Positioning in Very Low Birth Weight Infants. J Obstet Gynecol Neonatal Nursing. 2002;31:138-46.

26. Van Sleuwen BE, Engelberts AC, BoereBoonekamp MM, Kuis W, Schulpen TWJ, L'Hoir MP. Swaddling: A Systematic Review. PEDIATRICS. 2007; 120(4):1097-1106. http://pediatrics.aappublications. org/content/120/4/e1097. Accessed Oct 2007.

27. Robert D. White. Recommended Standards for Newborn ICU Design. Report of the Eighth Census Conference on Newborn ICU Design. Committee to Establish Recommended Standards for Newborn ICU Design. Clearwater Beach, FL. 2012.

28. Philbin MK. The influence of auditory experience on the behaviour of preterm newborns. J Perinatol. 2000;20:77-87.

29. Conde-Agudelo A, Belizan JM, Diaz-Rossello J. Kangaroo mother care to reduce morbidity and mortality in low birthweight infants. Cochrane Database Syst Rev. 2011;3. http://www.cochrane.org. Accessed 2011.

30. Mori R, Khanna R, Pledge D, Nakayama T. Meta-analysis of physiological effects of skin-to-skin contact for newborns and mothers. Pediatr Int. 2010; 52(2):161-70.

31. Als H, Gilkerson L, Duffy FH, McAnulty GB, Buehler DM, VandenBerg $\mathrm{K}$,et al. A three-center, randomized, controlled trial of individualized develop- 
mental care for very low birth weight preterm infants: medical, neurodevelopmental, parenting, and caregiving effects. J Dev Behav Pediatr. 2003;24:399-408.

32. Buehler DM, Als H, Duffy FH, McAnulty GB, Liederman J. Effectiveness of individualized developmental care for low-risk preterm infants: behavioral and electrophysiological evidence. Pediatrics. 1995; 96:923-32.

33. Symington A, Pinelli J. Developmental care for promoting development and preventing morbidity in preterm infants. Cochrane Database Syst Rev. 2001;4. http://onlinelibrary.wiley.com/doi/10.1002/14651858. CD001814.pub2/full. Accessed 2001.

34. Peters KL, Rosychuk RJ, Hendson L, Cote JJ, McPherson C, Tyebkhan JM. Improvement of Shortand Long-Term Outcomes for Very Low Birth Weight Infants: Edmonton NIDCAP Trial. Pediatrics. 2009;
124:1009-1020. https://www.ncbi.nlm.nih.gov/ pubmed/19786440. Accessed 2009.

35. Als H, Duffy FH, McAnulty G , Butler SC, Lightbody L, Kosta S, et al. NIDCAP improves brain function and structure in preterm infants with severe intrauterine growth restriction Journal of Perinatology. 2012;32:797-803.

36. Kleberg A, Westrup B, Stjernqvist K. Developmental outcome, child behaviour and mother-child interaction at 3 years of age following Newborn Individualized Developmental Care and Intervention Program (NIDCAP) intervention. Early Human Development. 2000;60:123-135.

37. Kleberg A, Hellstrom-Westas L, Widstrom A. Mothers' perception of Newborn Individualized Developmental Care and Assessment Program (NIDCAP) as compared to conventional care. Early Human Development. 2007;83:403-411.

Received: 2017-01-19 